\title{
Uso del modelo EFQM como contraste del nivel de gestión de la calidad en los servicios deportivos municipales
}

\author{
Using the EFQM model in contrast with the level of \\ quality management in municipal sports services
}

\author{
A. Martínez-Moreno y A. Díaz Suárez
}

Universidad de Murcia, Campus de Excelencia Internacional Regional “Campus Mare Nostrum” (España).

\begin{abstract}
Resumen: Las nuevas demandas de los usuarios han presionado al sector deportivo a emprender profundas remodelaciones en sus estructuras, siendo primordial la calidad en el servicio. En este estudio, se evalúa a través del Modelo Europeo de Excelencia el nivel de calidad de diez ayuntamientos de la Región de Murcia con menos de 10.000 habitantes. La muestra formada por 66 trabajadores, 41 hombres $(62.1 \%)$ y 25 mujeres (37.9\%). Validez de constructos análisis factorial confirmatorio, fiabilidad Alfa de Cronbach (.098), nivel de significación de $\mathrm{p} \leq .05$. El perfil de los servicios deportivos permite conocer sus puntos fuertes: resultados clave 97 puntos $(64.4 \%)$ sobre 150 del modelo y sus puntos débiles: alianzas y recursos 45 puntos (50.3\%) de los 90 máximo del modelo. Globalmente consiguen 580 puntos (57.1\%) de los 1000 que se pueden alcanzar, por lo que su nivel de calidad, según el modelo, es aceptable.

Palabras clave: Calidad, Gestión deportiva, Modelo EFQM, servicios deportivos municipales, evaluación.
\end{abstract}

Abstract: The new demands of the users have pressed to the sports sector to undertake deep remodelings in his structures, being basic the quality in the service. In this study, it is evaluated, through the European Model of Excellence, the quality level of ten town halls of the Region of Murcia, with less than 10.000 inhabitants. The sample was formed by 66 workers, 41 men $(62.1 \%)$ and 25 women $(37.9 \%)$. The statistical techniques used were construct validity, confirmatory factorial analysis, reliability by Cronbach's alpha (.098), level of significance of $\mathrm{p}=.05$. The profile of the sports services allows to know their strong points: key results 97 points $(64.4 \%)$ out of 150 of the model and their weak points: alliances and resources 45 points $(50.3$ $\%)$ out of 90 maximum of the model. Globally they obtained 580 points $(57.1 \%)$ out of the 1000 that could be reached, therefore their level of quality, according to the model, is acceptable.

Keywords: Quality, Sports Management, Model EFQM, municipal sports services, evaluation.

\section{Introducción}

En las últimas décadas los gobiernos, en parte debido a la recesión económica así como a la sobredimensión de personal e infraestructuras de todo tipo, han comenzado a plantearse la necesidad de reformar las estructuras organizativas y sistemas de gestión. Uno de los pilares ha sido la calidad, primero, por la necesidad de garantizar un producto adecuado, después, para asegurar su propia supervivencia, buscando la fidelización y adhesión de los usuarios, así como estrategia de posicionamiento en el mercado, buscando de este modo una ventaja competitiva que les diferencie de los demás.

El sector servicios, no ha sido ajeno a la implantación de técnicas de evaluación y de mejora de la calidad que ha traído como consecuencia inmediata un rápido desarrollo de indicadores y de métodos de evaluación. Diferentes razones han propiciado este interés: el compromiso ético de buscar lo mejor para el usuario (asegurando la efectividad), razones de índole organizativa servicios deportivos municipales más dinámicos y cercanos a los clientes externos, necesidad de contener el gasto creciente (con una clara preocupación por la eficiencia), junto con el compromiso social de ser sostenibles y

Dirección para correspondencia [Correspodence address]: Alfonso Martínez-Moreno. Universidad de Murcia. E-mail: almamo@um.es ayudar a la sociedad. En interés y preocupación por la calidad en la prestación de los servicios públicos es una constante de todos los implicados que intervienen en la servucción (proceso de creación de los servicios) en empresas públicas y privadas así como en los servicios deportivos (usuarios, clientes internos, gestores o políticos), tanto por razones éticas como por otras razones de interés particular, de índole económica y de efectividad de los procesos. Por esta razón, no siempre coincidirán los puntos de vista sobre las cualidades de un buen servicio. Esto no es un problema o una contradicción, sino percepciones diferentes acerca de qué es la calidad de los servicios deportivos municipales. Hay relativamente pocos estudios desde la perspectiva del proveedor de servicios algunos han analizado la gestión y las políticas llevadas a cabo por los Servicios Deportivos Municipales en adelante (SDM) (Gharakhania, Rasouliband Babakhanic, 2011).

El objeto de estudio es contrastar el nivel de gestión de la calidad de los Servicios Deportivos Municipales (SDM) de 10 ayuntamientos con menos de 10.000 habitantes en adelante $(<10.000 \mathrm{hab})$ mediante la autoevaluación de sus clientes internos usando el cuestionario del Modelo de la European Foundation for Quality Management en adelante (EFQM), contextualizado a los SDM con objeto de representar y re- 
flejar las singularidades y peculiaridades que caracterizan a los mismos, respetando número de criterios y de ítems que indica el Modelo.

\section{Los servicios deportivos municipales de calidad}

Mientras la calidad es pertinente tanto en bienes como a servicios, los servicios plantean cuestiones únicas en la definición de la calidad y en la realización de iniciativas de calidad (Grönroos, 1984, 1988; Lehtinen y Lehtinen, 1991; Zeithaml, Parasuraman, y Berry, 1988). Los servicios se diferencian de productos manufacturados en términos de como ellos, son producidos, consumidos, y evaluados. Más expresamente, los servicios son intangibles, no se puede tocar ni almacenar, durante su prestación no puede ser percibido físicamente. La inseparabilidad no pueden separarse de quien los presta o crea, la servucción va unida al consumo imposibilidad de separar la producción del consumo. La variabilidad, la prestación de un servicio, nunca se producirá de la misma manera. La caducidad, si no se consumen en el momento de ser producidos, no se pueden almacenar. Estos atributos destacan la idea que las interacciones entre el cliente y empleado del servicio son críticas a la producción y el consumo de un servicio.

Para De la Plata (2001), en la organización del servicio municipal se pueden distinguir tres etapas, primera predemocrática, donde la gestión directa por la propia entidad local era prácticamente indiscutida, a través de una Concejalía de Deportes con poco peso específico normalmente, una segunda transición democrática, en la que el organismo autónomo local (bajo el nombre, acertado o no, de Patronatos, Fundaciones o Institutos de Deportes) recoge todas las competencias deportivas, creando una estructura técnica que en algunos casos ha llegado a desbordarse y una tercera que podríamos denominar consolidación democrática, donde nos encontramos, en la que algunos autores (Delgado y Prieto, 1997; Pérez, 2000; De la Plata, 2001), tratan de utilizar plenamente los instrumentos legales al alcance, estableciendo un sistema mixto, donde tienen cabida varios modos de gestión según la actividad que se trate, pero alejándose de la uniformidad imperante, con mayor flexibilidad, teniendo la gestión indirecta como instrumento muy valorado.

En la gestión local, resulta muy difícil definir un modelo único de actuación deportiva, ya que la autonomía de cada Ayuntamiento, las distintas realidades, el ámbito competencial, la cultura deportiva y los recursos con que cuenta cada localidad, en definitiva, lo que se viene denominando el Sistema Deportivo Local, impide un planteamiento común (Garde, 2003).

Las estructuras organizativas municipales, en municipios de un tamaño poblacional mediano o grande suelen crear un organismo autónomo local con diferentes nombres: Patronato Municipal de Deportes, Instituto Municipal de Deportes,
Fundación Deportiva Municipal, etc., de carácter administrativo, al objeto de gestionar el área deportiva directamente, pero mediante descentralización funcional. Aunque es un organismo autónomo, normalmente tiene una vinculación directa con el Concejal de Deportes o con el Alcalde. Se suelen estructurar en tres áreas distintas: por un lado el personal de administración, por otro el personal encargado de impartir los servicios deportivos que se ofertan, por último, el personal de mantenimiento.

Establecer un modelo de función municipal en el deporte, como en cualquier otro ámbito, para todos los ayuntamientos, sería una osadía. La autonomía con que cuenta el municipio, propicia precisamente, que cada cual pueda adaptar su papel a su realidad concreta. Por tanto, el primer paso para discernirlo, consiste en realizar un profundo análisis sobre el Sistema Deportivo Territorial del Municipio. Ya que muchos, y a trepidante ritmo, han sido los cambios experimentados por la sociedad española después de la Constitución Española de 1978. El artículo 43.3 de la misma, fue punto de arranque para que las instituciones abordaran con entusiasmo la promoción de la convivencia social a través de la actividad físico-deportiva.

El papel de los municipios en vistas de la actual y próxima década debe orientarse a cumplir las siguientes funciones (1) administradora de los recursos disponibles con eficacia y eficiencia, (2) coordinadora con un abandonando progresivo del protagonismo e implicar más a las asociaciones y agentes sociales en la gestión, (3) reguladora de la gestión y reglamentos del uso de instalaciones y espacios deportivos con la participación de los ciudadanos, (4) integradora posibilitando la práctica deportiva a todos los ciudadanos, como mejora de su calidad de vida, (5) inversora para tener una red de equipamientos públicos que permitan el acceso a todos los ciudadanos a la práctica deportiva y (6) planificadora: definir unos objetivos concretos y diseñar las estrategias a seguir para conseguirlos, con los medios y recursos necesarios estableciendo unos plazos de tiempo determinados para su consecución (Delgado, 2005).

Los Sistemas de Calidad son herramientas que se centran en garantizar que lo que ofrece una entidad cumpla unas especificidades establecidas previamente por dicha entidad y el cliente. La calidad según Gutiérrez, Vázquez y Cuesta (2010) es un elemento esencial para la mejora de los recursos humanos, la productividad, la adaptación de las necesidades sociales. Araújo y Sampaio (2014) mostraron cómo las organizaciones que han alcanzado un reconocimiento EFQM han progresado a través de un proceso de desarrollo más maduro y estadios avanzados.

La calidad del servicio se descompone en tres variables para Grönroos (1994) (1) la calidad técnica (o del resultado), entendida como el resultado del servicio para el cliente, el "qué", (2) la calidad funcional (o del proceso), que es la forma 
en la que el cliente recibe el servicio, es decir, el "cómo", (3) la imagen del servicio, como filtro que matiza las valoraciones efectuadas por el cliente sobre la calidad técnica y la calidad funcional.

La diferencia entre Calidad del servicio y satisfacción no está del todo clara, si bien se ha generalizado la idea de que la primera se obtiene tras una larga y completa evaluación, mientras que la segunda es la medida de una transacción específica.

Es significativo, que la satisfacción con el uso del servicio deportivo y los programas ofertados puedan influir en su práctica y no al revés, ya que la actitud positiva hacia la práctica de la Actividad Física no presupone satisfacción en el uso del servicio y es que la satisfacción del consumidor influye en sus intenciones futuras y en sus actitudes (Oliver, 1980).

El producto y las evaluaciones de calidad del servicio están en su mayoría basadas en un mecanismo de procesamiento cognitivo (Vida y Reardon, 2008). Por otro lado, la satisfacción es un resultado psicológico derivado de la experiencia (Lee, Petrick y Crompton, 2007). Por lo tanto, el subyacente mecanismo de procesamiento para evaluar la calidad y la satisfacción son distintos (Zabkar, Brencic y Dmitrovic, 2009).

\section{El Modelo de la European Foundation for Quality Management (EFQM)}

El Modelo se fundamenta en que los resultados excelentes con respecto al rendimiento de la organización, a los clientes, a las personas y a la sociedad mediante un liderazgo que dirija e impulse la política y estrategia, las personas de la organización, las alianzas y recursos, y los procesos. Los resultados en los clientes, resultados en las personas, gestión de las personas y resultados en la sociedad, se consiguen por medio del liderazgo que a través de unos procesos establecidos conduce una política y estrategia, gestiona unas personas, y se sirve de unos recursos y alianzas, llevando finalmente a la excelencia en los resultados de la Organización. Cada uno de sus nueve elementos (criterios) del modelo se evalúa (otorgando hasta un máximo de puntos para cada criterio) y ponderan (porcentualmente) para determinar el progreso de la organización hacia la excelencia (EFQM, 2010). Agentes "criterios que cubren lo que es hecho por la organización y los criterios de "resultados", cubren lo que una organización logra (Sadikoglu y Olcay, 2014).

El modelo de la EFQM se basa en la autoevaluación un ejercicio mediante el cual la organización se compara frente a un modelo de excelencia y obtiene cuáles son sus puntos fuertes y áreas de mejora en relación con dicho modelo. Es un excelente punto de partida para el proceso de planificación empresarial, ya que partiendo de los puntos fuertes y las áreas de mejora permite identificar los planes de mejora que serán parte integral de los planes empresariales a corto, medio y largo plazo que deben hacer a la organización más competitiva. Ventajas del modelo EFQM como herramienta de autoevaluación (1) participación de todos los actores en los ciclos de evaluación y proyectos de mejora, (2) considerar todos los aspectos, para el rendimiento organizacional, (3) apto para ser aplicado y adaptado (4) compromete a cada uno de los integrantes para mejorar su ambiente laboral y su relación con el usuario.

El Modelo lo crearon en Europa 14 empresas líderes, en septiembre del año 1988 formaron la Fundación Europea para la Gestión de la Calidad Total (EFQM), estableciendo en 1991 el Premio Europeo a la Calidad (que se pretende equivalente al premio Baldrige americano). En 1994, un grupo de trabajo adaptó el modelo al Sector Público: atención sanitaria, educación, ayuntamientos y otros sectores públicos, publicando actualizaciones periódicas (1999, 2003, 2010).

\section{Método}

\section{Participantes}

La muestra está formada por un total de 66 trabajadores directos e indirectos de diez SDM de la Región de Murcia ubicados en Ayuntamientos < 10000 hab, de los que 41 son varones $(62.1 \%)$ y 25 mujeres $(37.9 \%)$ edad \pm 31 , ańos. Los participantes pertenecen a todos los grupos y niveles profesionales de la Administración Local (personal de limpieza, mantenimiento, administrativos, auxiliares administrativos, técnicos deportivos, coordinadores, gerentes, etc.) exceptuando los cargos políticos. Quedando distribuida en relación a su nivel de estudios de la siguiente forma: dos licenciados en Educación Física (3\%), siete Diplomados-ingenieros (11\%), nueve Ciclos Formativos/FP (14\%), 14 con Bachiller (21\%), tres con estudios primarios (5\%), 12 Maestros Educación Física (18\%), seis licenciados en otras titulaciones (9\%), cuatro con Ciclos Formativos en Actividad Física y Deportes (6\%) un administrativo (2\%).

\section{Instrumento}

Se partió del cuestionario del EFQM elaborado por (European Foundation for Quality Management, 2003), el cual está constituido por nueve factores, para el Sector Público y las Organizaciones del Voluntariado. Contextualizado a los Servicios Deportivos Municipales con objeto de representar y reflejar las singularidades y peculiaridades que caracterizan a los mismos, respetando número de criterios y de ítems que indican el Modelo. La base del cuestionario es el utilizado por Martínez-Moreno, López y López (2006) denominado Servicios Municipales de Calidad (SERMUCAL).

El cuestionario está compuesto por un total de 116 ítems de los que 11 son datos sociodemográficos. El resto de ítems 
son de respuesta cerrada, con una escala tipo Likert que va desde 1. Nada o muy poco, 2. Poco, 3. Mucho, hasta 4. Del todo, quedando distribuidos de la siguiente forma: Liderazgo con 11 ítems, Política y Estrategia con 12 ítems, Gestión de las personas con 11 ítems, Alianzas y Recursos con 12 ítems, Procesos con 11 ítems, Resultados en los usuarios-clientes externos con 14 ítems, Resultados en las personas con 12 ítems, Resultados en la sociedad con 7 ítems, Resultados Clave con 15 ítems, distribución indicada por el Modelo EFQM.

\section{Procedimiento}

Se realizó un muestreo no probabilístico intencional (Sierra, 2007), concertando una reunión con cada uno de los nueve SDM analizados, mientras los clientes internos-trabajadores rellenaban el cuestionario autoadministrado, estaba presente un investigador formado a tal efecto, para aclarar las dudas que pudieran surgir.

\section{Análisis de datos}

Con el objetivo de poder comparar los valores de las diferentes sub-escalas de cada uno de los factores del modelo EFQM, se utilizó la prueba paramétrica de t de Student para muestras apareadas. Para el cálculo de la validez de constructo se utilizó el análisis factorial confirmatorio (Thomsom, 2004). Como estadígrafo de ajuste se utilizó el RMSEA (root mean square error of aproximation), el índice de bondad de ajuste (GFI). La fiabilidad del modelo EFQM se calculó mediante el análisis de la consistencia interna, para lo cual se utilizó el coeficiente Alfa de Cronbach, el cual debe interpretarse como un indicador de la consistencia interna de los ítems, ya que se calcula a partir de la covarianza entre ellos. Los índices de fiabilidad situados alrededor de .70 sugerirían que existe una adecuada consistencia interna (Celina y Campo, 2005). Para el análisis de los datos se utilizó el programa estadístico SPSS 17.0, en su versión para Windows. Los análisis estadísticos se han realizado con un nivel de significación de $\mathrm{p} \leq .05$. Para el análisis factorial confirmatorio se utilizó el programa, LISREL, versión 8.54 .

\section{Resultados}

Las puntuaciones que se obtienen respecto al bloque de agentes facilitadores son; Criterio 1. Liderazgo con 63 puntos
(62.6\%) apreciando diferencias estadísticamente significativas entre los subcriterios la y $1 \mathrm{e}(\mathrm{t} 65=2.238, \mathrm{p}=.029), 1 \mathrm{~b}$ y $1 \mathrm{e}$ (t63 = 2.399, p=.019), 1c y 1e (t62 = 2.672, p=.010). Criterio 2. Política y Estrategia, alcanzan 40 puntos (50.6\%) de los 80 máximos del Modelo, estimando diferencias estadísticamente significativas entre los subcriterios $2 \mathrm{a}$ y $2 \mathrm{~b}(\mathrm{t} 62=-229, \mathrm{p}=$ .029), $2 \mathrm{~b}$ y $2 \mathrm{~d}(\mathrm{t} 63=3.686, \mathrm{p}=.001), 2 \mathrm{c}$ y $2 \mathrm{~d}(\mathrm{t} 60=3.120$, $\mathrm{p}=.003)$. Criterio 3. Gestión de las personas, obtienen de los 90 posibles $50(55,1 \%)$ contrastando diferencias estadísticamente significativas entre los subcriterios $3 \mathrm{a}$ y $3 \mathrm{c}(\mathrm{t} 61=$ 2.043, $\mathrm{p}=.045), 3 \mathrm{a}$ y $3 \mathrm{~d}(\mathrm{t} 61=4.149, \mathrm{p}=.001), 3 \mathrm{~b}$ y $3 \mathrm{~d}(\mathrm{t} 61$ $=4.414, \mathrm{p}=.001), 3 \mathrm{c}$ y $3 \mathrm{~d}(\mathrm{t} 59=3.255, \mathrm{p}=.002), 3 \mathrm{~d}$ y $3 \mathrm{e}(\mathrm{t} 61$ $=-2.582, \mathrm{p}=.012)$. Criterio 4. Alianzas y Recursos consiguen $45(50,3 \%)$ de los 90 puntos que marca el modelo, existiendo diferencias estadísticamente significativas entre los subcriterios 3a y 3c $(\mathrm{t} 61=2.043, \mathrm{p}=.045)$, 3a y $3 \mathrm{~d}(\mathrm{t} 61=4.149$, $\mathrm{p}=.001), 3 \mathrm{~b}$ y $3 \mathrm{~d}(\mathrm{t} 61=4.414, \mathrm{p}=.001), 3 \mathrm{c}$ y $3 \mathrm{~d}(\mathrm{t} 59=3.255$, $\mathrm{p}=.002)$, $3 \mathrm{~d}$ y $3 \mathrm{e}(\mathrm{t} 61=-2.582, \mathrm{p}=.012)$. Criterio 5 . Procesos obtienen 72 puntos $(51,4 \%)$ de los 140 que marca el modelo, encontrando diferencias estadísticamente significativas entre los subcriterios $5 \mathrm{a}$ y $5 \mathrm{~b}(\mathrm{t} 60=-4.882, \mathrm{p}=.001), 5 \mathrm{~b}$ y $5 \mathrm{c}(\mathrm{t} 60=$ $3.502, \mathrm{p}=.001), 5 \mathrm{~b}$ y $5 \mathrm{~d}(\mathrm{t} 60=5.043, \mathrm{p}=.001), 5 \mathrm{~b}$ y $5 \mathrm{e}(\mathrm{t} 61=$ 4.321, $\mathrm{p}=.001), 5$ c y $5 \mathrm{~d}(\mathrm{t} 60=2.080, \mathrm{p}=.042)$.

Los resultados del segundo boque denominado agentes resultados son; Criterio 6. Resultados Clientes externos con 126 puntos $(63,2 \%)$ de los 200 que marca el modelo, no existen diferencias estadísticamente significativas, entre los subcriterios 6a y 6b. Criterio 7. Resultados en las personas obtienen 54 puntos $(60,2 \%)$ de los 90 máximos del modelo, no existen diferencias estadísticamente significativas según los datos, entre los subcriterios $7 \mathrm{a}$ y $7 \mathrm{~b}$. Criterio 8 consiguen 34 puntos $(56.9 \%)$ de los 60 máximos del modelo, existen diferencias estadísticamente significativas, entre los subcriterios $8 \mathrm{a}$ y $8 \mathrm{~b}(\mathrm{t} 63=4.440, \mathrm{p}=.001)$. Criterio 9. Resultados clave, obtienen 97 puntos $(64,4 \%)$ de los 150 máximos del modelo, existen diferencias estadísticamente significativas, entre los subcriterios 9a y $9 \mathrm{~b}$ ( $\mathrm{t} 58=-4.648, \mathrm{p}=.001)$.

Una vez analizados todos los criterios, mostramos en la figura 1, cual es el perfil de los SDM $<10000$ hab. Alcanzan 580 puntos (57.1\%) de los 1000 máximos que marca el Modelo. Es el criterio 9. Resultados Clave, con 97 puntos (64.4\%) el que obtiene la puntuación más alta. Mientras que obtiene menor valoración 45 puntos (50.3\%), el criterio 4 . Alianzas y Recursos. 
Figura 1. Perfil de los SDM. Ayuntamientos $<10.000$ hab.

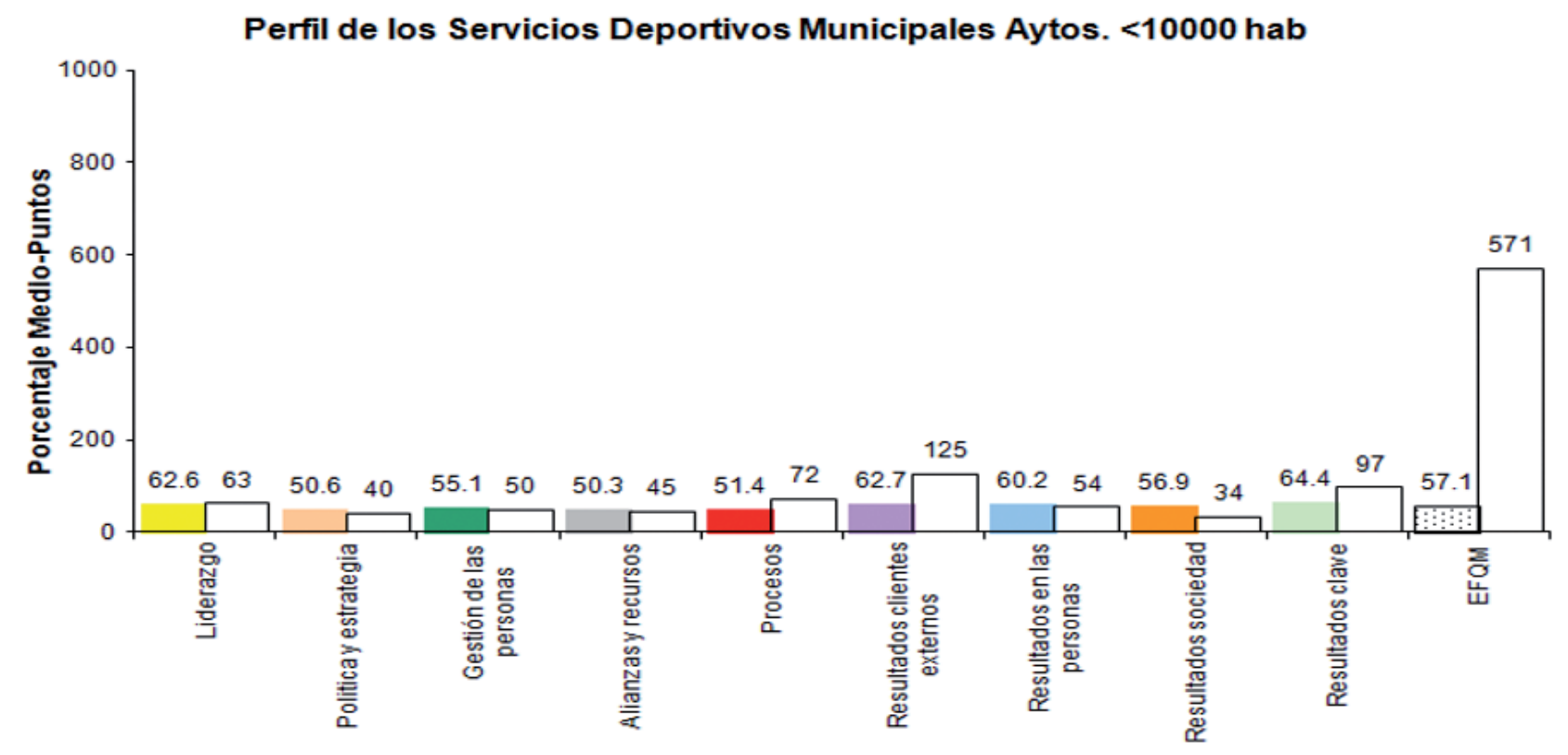

\section{Discusión}

Los 10 ayuntamientos con $<10000$ hab., superan ampliamente en todos los criterios al Servicio de Deportes de la Universidad de Vigo, del estudio de Rial et al., (2004) así como al Servicio de Actividades Deportivas de la Universidad de Murcia, de la investigación de Martínez-Moreno, López y López (2006) también superan en todos los criterios, excepto en Alianzas y recursos que están en la misma línea (45-40) puntos respectivamente, al Servicio de Actividades Deportivas de la Universidad de Sevilla, de la investigación de Galán (2004). Obtiene resultados equivalentes en relación al Servicio de Promoción Deportiva de la Universidad Politécnica de Cartagena, de la investigación de Martínez-Moreno, López y López (2006) en los criterios Política y estrategia (40-49), Gestión de las personas (50-45) Resultados en las personas (54-55) Resultados en la Sociedad (34-40), obteniendo menor puntuación en Liderazgo, Alianzas y Recursos, Procesos y Resultados en la Sociedad, aunque los superan en el criterio Resultados en los clientes y Resultados Clave. Quedan por debajo en ocho criterios en relación al Servicio de Actividades Deportivas de la Universidad Católica San Antonio del estudio de Martínez-Moreno, López y López (2006) excepto en el criterio Resultados Clave superándolo en más de 10 puntos.

\section{Conclusiones}

Como conclusiones decir que los líderes de estos servicios de deportes con $<10.000$ hab., interactúan con clientes, asociados y representantes de la sociedad, mientras que, esos mis- mos líderes, no impulsan el cambio en la organización. Esta adecuada atención al usuario es uno de los aspectos que la gran mayoría de autores definen y defienden como elemento clave para alcanzar unos altos niveles de calidad en la gestión, tanto en servicios y empresas en general (Bitner, Booms y Mohr, 1994; Price, Arnold, y Terney, 1995), como en servicios y empresas deportivas en particular (Carver, 1997). La política y estrategia se basa en la información de los indicadores de rendimiento, la investigación, el aprendizaje y las actividades externas, aunque no se comunica y despliega mediante un esquema de procesos clave. Rubio (2008), indica que tiene una finalidad funcional e instrumental, dar información apoyo individual, formación y canalizar los intereses del colectivo. Existe poco dialogo entre las personas y la organización, sin embargo es aceptable, la planificación, gestión y mejora de los recursos humanos. Es escasa la gestión de la información y del conocimiento, mientras que la gestión de las alianzas es aceptable. Para alcanzar valores elevados de calidad, tanto en servicios en general (Jareńo, 2008), como en servicios deportivos en particular (Congreso Panamericano de Educación Física, 2005), es necesario compartir experiencias, conocimientos, etc., con otras unidades. Introducen las mejoras necesarias en los procesos mediante la innovación, a fin de satisfacer plenamente al cliente y otros grupos de interés, generando cada vez mayor valor debiendo mejorar la gestión y mejora de las relaciones con los clientes. Tienen pocas quejas y reclamaciones mientras que es escasa la accesibilidad y transparencia. Están motivados y satisfechos indicando que su productividad no es la adecuada La imagen general que proyectan y la implicación en su entorno son al- 
tas, aunque carecen de certificaciones, premios públicos que lo corroboren. En relación a los Resultados Calve, los costes de mantenimiento y proyectos son equilibrados, aspecto clave para lograr unos valores elevados de calidad (Chelladurai y Haggerty, 1991) siendo deficitarios el rendimiento de los procesos requiriendo revisión y actualización de los mismos.
Siendo los sistemas de calidad elementos imprescindibles en la gestión de la calidad y por ende de la Excelencia, ofreciendo la posibilidad a las organizaciones de ser proactivas ante sus clientes externos diferenciándose de la competencia y ofertando los servicios adecuados a cada usuario.

\section{Referencias Bibliográficas}

1. Araújo, M., y Sampaio, P. (2014). El camino a la excelencia de los portugueses de las organizaciones reconocidas por el modelo EFQM. Gestión de la Calidad Total y la excelencia empresarial, Vol. 25, No 5-6, pp. 427438.

2. Bitner, M.J., Booms, B.H., y Mohr, L.A. (1994). Critical service encounters: The employee's viewpoint. Journal of Marketing, 58, 95-106.

3. Carver, J. (1997). Boards that make a difference: A new design for leadership in nonprofit and public organizations ( $2^{\text {nd }}$ ed.). San Francisco: JosseyBass.

4. Celina, H. y Campo, A. (2005): "Aproximación al uso del coeficiente Alfa de Cronbach”. Revista Colombiana de Psiquiatria, 34, 572-580.

5. Chelladurai, P., y Haggerty, T. R. (1991). Measures of organizational effectiveness of Canadian national sport organizations. Canadian Journal of Sport Sciences, 16,126-33.

6. Congreso Panamericano De Educación Física XIX. (2005) Una visión hacia la Educación Física, El Deporte y la Recreación. "Calidad y Equidad". Recuperado de http://www.sportsalut.com.ar/articulos/educacion_fisica/nuevo. pdf

7. De la Plata, N. (2001). Los servicios públicos deportivos. Madrid: CEES Ediciones.

8. Delgado, A. y Prieto, G. (1997). Introducción a los métodos de investigación en la psicología. Madrid: Pirámide.

9. Delgado, C. (2005). Dirección y gestión del deporte municipal. Recuperado de http://www.femp.esindex.phpfempareas_de_gesi_nservcios_ localeseducaci_n_deportes_cultura_y_juventuddeportedocumentos_ de_inter_s Ponencias Curso (para la web)

10. European Foundation for Quality Management [EFQM]. (2010). Introducing the EFQM Excellence Model 2010. [online] EFQM. Recuperado de: http://www.efqm.org/en/PdfResources/EFQMModel_Presentation.pdf> [fecha de consulta: enero de 2010].

11. European Foundation For Quality Management. (2003). Modelo EFQM de Excelencia. Versión para el Sector Público y las Organizaciones del Voluntariado. Madrid: Club Gestión de la Calidad.

12. Galán, M. (2004). Informe de la evaluación externa del Servicio de Actividades Deportivas de la Universidad de Sevilla. Recuperado de: http://www. us.es/sadus/documentos/ INFORME_EVAL_SADUS. pdf.

13. Garde, A. (2003). Funciones y Directrices en la Gestión Local. Recuperado de http://www.depormadrid.com/depor11/reportajes/reportajes. htm

14. Gharakhania, M., Rasoulib, S. and Babakhanic, M. (2011). A robust LINMAP for EFQM self assessment. Management Science Letters, 1 (2011), pp. 213-222, doi: 10.5267/j.msl.2010.02.001.

15. Grönroos, C. (1984). A service quality model and its marketing implication. European Journal of Marketing, 18(4), 36-44 0200

16. Grönroos, C. (1988). Service quality: The six criteria of good perceived service. Review of Business, 9 (3), 10-13. 0116

17. Grönroos, C. (1994). Marketing y Gestión de Servicios. Madrid: Díaz de Santos.
18. Gutiérrez, P., Vázquez, J. L., y Cuesta, P. (2010). Valoración de los factores determinantes de la calidad del servicio público local : un análisis de la percepción de los ciudadanos y sus repercusiones sobre la satisfacción y credibilidad. Innovar, 20 (36), 139-156.

19. Jareño, O. (2008). Alianzas Estratégicas. La Herramienta para Fortalecer la Empresa. Recuperado de http://www.puromarketing.com/13/5119/ alianzas-estrategicas-herramienta- para-fortalecer-empresa.html

20. Lee, S. Y., Petrick, J. F., y Crompton, J. (2007). The roles of quality and intermediary constructs in determining festival attendees behavioral intention. Journal of Travel Research, 45(4), 402-412.

21. Lehtinen, U., y Lehtinen, J.R. (1991). Two approaches to service quality dimensions. The Service Industries Journal, 11, 287-303. 0114

22. Martínez-Moreno, A.; López, M.A. y López, J.M. (2006). Planificación de la calidad en Actividad Física. Ed. Servicio de Publicaciones. Universidad de Murcia. Instituto Propio de Ciencias del Deporte, Granada.

23. Oliver, R.L. (1980). A cognitive midel of the antecedents and consequences of satisfaction decisions. Journal Marketing Research, 17, 460469.

24. Pérez, M. (2000). Perspectivas de la gestión municipal y modelos de gestión. En: Actas del $1^{\mathrm{er}}$ Congreso de Gestión Deportiva de Cataluña (pp. 25-129). Barcelona: Inde.

25. Price, L.L., Arnold, E.J. y Terney, P. (1995). Going to extremes: Managing service encounters and assessing provider performance. Journal of Marketing, 59, 83-97.

26. Rial, J., Loureiri, G., Pérez, L., Alberte, R., Rodríguez, E., Sanjorge, A., Lago, A., Amosa, E., Iglesias, C., Rodríguez, D. y Álvarez, E. (2004). II Plan de la calidad de las Universidades. Informe de autoevaluación Servicio de Deportes. Recuperado de: http://webs.uvigo.eswebcalidadarea _calidad informes IIPCUServiciosinf autoav_Deportes.pdf.

27. Rubio, J. (2008). Espacios sociales de participación: las asociaciones y los grupos de autoayuda. Nómadas. Revista Crítica de Ciencias Sociales y Jurídicas, 18, 2. Recuperado de http://www.ucm.es/info/nomadas /18/ fjrarribas .pdf

28. Sadikoglu, E. y Olcay, H. (2014). Los efectos de las prácticas de gestión de la Calidad Total en el rendimiento y los motivos y las barreras para la GCT prácticas en Turquía. Los avances en las ciencias de la Decisión, 2014.

29. Sierra, R. (2007). Tesis doctorales y trabajos de investigación cientifica. Ed. Thomson Editores. Paraninfo, S.A.Madrid.

30. Thomsom, B. (2004). Exploratory and confirmatory factor analysis. Washington: American Psychological Association.

31. Vida, I. y Reardon, J. (2008). Domestic consumption: rational, affective or normative choice. Journal of Consumer Marketing, 25(1), 34-44.

32. Zabkar, V., Brencic, M.M. y Dmitrovic, T. (2009). Modelling perceived quality, visitor satisfaction and behavioural intentions at the destination level, Tourism Management, doi:10.1016/j.tourman.2009.06.005

33. Zeithaml, V.A., Parasuraman, A. y Berry, L.L. (1988). Comunication and Control Process in the Delivery of Service Quality. Journal of Marketing, 52, 35-48. 